\title{
MEROMORPHIC SOLUTIONS OF $\boldsymbol{P}_{4,34}$ AND THEIR VALUE DISTRIBUTION
}

\author{
Ewa Ciechanowicz and Galina Filipuk
}

\author{
University of Szczecin, Institute of Mathematics \\ ul. Wielkopolska 15, 70-451 Szczecin, Poland; ewa.ciechanowicz@usz.edu.pl \\ University of Warsaw, Department of Mathematics, Informatics and Mechanics \\ Banacha 2, 02-097, Warsaw, Poland; filipuk@mimuw.edu.pl
}

\begin{abstract}
The unified equation $P_{4,34}$ is closely related to the well-known Painlevé equations $P_{2}$ and $P_{4}$. We discuss various properties of solutions of $P_{4,34}$, including one-parameter families of solutions, Bäcklund transformations, regular systems for expansions around zeros and poles and value distribution. In particular, we give estimates of defects and multiplicity indices of transcendental meromorphic solutions of this equation. Moreover, we study solutions of $P_{4,34}$ from the perspective of Petrenko's theory, which is also new for $P_{2}, P_{4}$ and $P_{34}$. We give estimates of deviations and analyse the sets of exceptional values in the sense of Petrenko for equations $P_{2}, P_{4}, P_{34}$ and the unified equation $P_{4,34}$.
\end{abstract}

\section{Introduction}

In this paper we begin with elementary notions of value distribution theory paying special attention to the notion of deficiency. Then we present results of Clunie type and Mohon'ko-Mohon'ko type. Next we give an overview of the known results concerning value distribution of the Painlevé equations $P_{2}$ and $P_{4}$ and we move on to new results stated for $P_{2}, P_{4}$ and the unified equation $P_{4,34}$. The paper ends with the Appendix, where further properties of $P_{4,34}$ are discussed. Some of the results given there are new and, to our knowledge, did not appear in any paper. Thus we present them to make the description of properties of $P_{4,34}$ complete.

\section{Preliminaries}

Let us start with the basic results of Nevanlinna theory. We apply the standard notations [9]. The following theorem is known as the first main theorem of Nevanlinna.

Theorem 1.1. [19] For any function $f$ meromorphic in the disc $|z|<R \leq \infty$ the equality

$$
m(r, a, f)+N(r, a, f)=T(r, f)+\phi(r, a),
$$

holds for each $a \in \overline{\mathbf{C}}$, where $|\phi(r, a)| \leq \log ^{+}|a|+|\log | c||+\log 2$ and $c$ is the first nonvanishing coefficient of the Laurent expansion of $f-a$ at zero.

In the standard way we define $\delta(a, f)$, the defect of $f$ at a value $a \in \overline{\mathbf{C}}$,

$$
\delta(a, f)=\liminf _{r \rightarrow \infty} \frac{m(r, a, f)}{T(r, f)}=1-\limsup _{r \rightarrow \infty} \frac{N(r, a, f)}{T(r, f)}
$$

doi:10.5186/aasfm.2016.4146

2010 Mathematics Subject Classification: Primary 30D35; Secondary 34M05, 34M55.

Key words: Meromorphic function, Painlevé equation, defect, deviation, ramification index. 
and $\vartheta(a, f)$, the index of multiplicity of a value $a$,

$$
\vartheta(a, f)=\liminf _{r \rightarrow \infty} \frac{N_{1}(r, a, f)}{T(r, f)},
$$

where $N_{1}(r, a, f):=N(r, a, f)-\bar{N}(r, a, f)$. If $\delta(a, f)>0$, then we say that the value $a$ is defective (in the sense of Nevanlinna), and if $\vartheta(a, f)>0$ we call $a$ a ramified value of $f$. Let us remind that as a result of the first and the second main theorems of Nevanlinna, the set $E_{N}(f)$ of defective values of a meromorphic function $f$ is at most countable and the following relations are true:

$$
0 \leq \delta(a, f)+\vartheta(a, f) \leq 1, \quad \sum_{a \in \overline{\mathbf{C}}}(\delta(a, f)+\vartheta(a, f)) \leq 2 .
$$

The order and the lower order of a meromorphic function $f$ are defined by

$$
\varrho(f):=\limsup _{r \rightarrow \infty} \frac{\log T(r, f)}{\log r}, \quad \mu(f):=\liminf _{r \rightarrow \infty} \frac{\log T(r, f)}{\log r} .
$$

If $\varrho(f)=\mu(f)$ then $f$ is called a function of a regular growth.

In 1969 Petrenko introduced the quantity (see [22])

$$
\beta(a, f)=\liminf _{r \rightarrow \infty} \frac{\mathcal{L}(r, a, f)}{T(r, f)}
$$

called deviation of a meromorphic function with respect to the value $a \in \overline{\mathbf{C}}$, where

$$
\mathcal{L}(r, a, f):= \begin{cases}\max _{|z|=r} \log ^{+}|f(z)| & \text { for } a=\infty, \\ \max _{|z|=r} \log ^{+}\left|\frac{1}{f(z)-a}\right| & \text { for } a \neq \infty .\end{cases}
$$

For $a \in \overline{\mathbf{C}}$ the inequality

$$
\delta(a, f) \leq \beta(a, f)
$$

follows easily from the definition of $\beta(a, f)$. Thus we have $E_{N}(f) \subset E_{\Pi}(f)$, where $E_{\Pi}(f):\{a \in \overline{\mathbf{C}}: \beta(a, f)>0\}$. For meromorphic functions of finite lower order we have upper bounds of deviations similar to those following from the first and second main theorems of Nevanlinna. Namely, it was proved by Petrenko in [22] that the set $E_{\Pi}(f)$ of exceptional values in the sense of Petrenko is at most countable and

$$
\beta(a, f) \leq B(\mu):= \begin{cases}\frac{\pi \mu}{\sin \pi \mu} & \text { if } \mu \leq 0.5 \\ \pi \mu & \text { if } \mu>0.5\end{cases}
$$

In [15] Marchenko and Shcherba proved that

$$
\sum_{a \in \overline{\mathbf{C}}} \beta(a, f) \leq 2 B(\mu)
$$

Both estimates are sharp. Let us mention here that the hypothesis that for entire functions of order $\varrho, 0.5 \leq \varrho<\infty$, the inequality $\beta(\infty, f) \leq \pi \varrho$ holds, was longstanding. Stated by Paley in 1932, it was proved in 1969 by Govorov [7].

Considering $f(z)=\exp (z)$ as an easy example, we have

$$
\varrho(f)=\mu(f)=1, \quad E_{N}(f)=E_{\Pi}(f)=\{0, \infty\},
$$

and for exceptional values:

$$
\delta(0, f)=\delta(\infty, f)=1, \quad \beta(0, f)=\beta(\infty, f)=\pi,
$$


SO

$$
\sum_{a \in \overline{\mathbf{C}}} \delta(a, f)=2 \quad \text { and } \sum_{a \in \overline{\mathbf{C}}} \beta(a, f)=2 \pi .
$$

In general the sets $E_{N}(f)$ and $E_{\Pi}(f)$ may differ. In 1987 Gol'dberg, Eremenko and Sodin proved that for any fixed positive number $\varrho$ and any given two sets $E_{1} \subset$ $E_{2} \subset \overline{\mathbf{C}}$, which are at most countable, there is a meromorphic function of order $\varrho$ such that

$$
E_{N}(f)=E_{1}, \quad E_{\Pi}(f)=E_{2}
$$

$[5,6]$. It is interesting to notice that such a function may even be a function of a regular growth. For functions of infinite order the deviation may be infinite and the set $E_{\Pi}(f)$ may be uncountable.

\section{Theorems of Clunie type and of Mohon'ko-Mohon'ko type}

In this section we recall the well-known theorems of Clunie and of Mohon'ko, Mohon'ko. We also present new theorems of similar character concerning Petrenko's deviation.

We use the notation $S(r, f)$ for $\phi:(0,+\infty) \rightarrow \mathbf{R}$ such that

$$
\phi(r)=o(T(r, f)), \quad r \rightarrow \infty, r \notin E,
$$

where $E$ is a set of finite linear measure. For meromorphic functions $g$, $f$ we say that $g$ is small with respect to $f$ if $T(r, g)=S(r, f)$.

In our considerations below we apply the following result, which is a more general version of the original lemma by Clunie (see [3]).

Theorem 2.1. [14] Let $f$ be a transcendental meromorphic solution of

$$
f^{n} P(z, f)=Q(z, f),
$$

where $n$ is a positive integer, $P(z, f), Q(z, f)$ are polynomials in $f$ and its derivatives with meromorphic coefficients $\left\{a_{\lambda}: \lambda \in I\right\}$, such that $m\left(r, a_{\lambda}\right)=S(r, f)$ for all $\lambda \in I$. If the total degree $d$ of $Q(z, f)$ as a polynomial in $f$ and its derivatives is $d \leq n$, then

$$
m(r, P(z, f))=S(r, f) .
$$

It should be noticed that in the original Clunie lemma, by assumption, the condition $T\left(r, a_{\lambda}\right)=S(r, f)(\lambda \in I)$ holds for the coefficients of the equation, while in Theorem 2.1 it is only assumed that the proximity function of the coefficients fulfills this condition.

We shall prove the following generalisation of the lemma on the logarithmic derivative for $\mathcal{L}\left(r, \infty, f^{(k)} / f\right)$ by induction.

Proposition 2.2. Let $f$ be a meromorphic function. Then, possibly except for $r$ in a set of finite linear measure, for $k=1,2, \ldots$ we have

$$
\mathcal{L}\left(r, \infty, \frac{f^{(k)}}{f}\right)=O(\log (r T(r, f))), \quad r \rightarrow \infty,
$$

where $f^{(k)}$ means the $k$-th derivative of $f$.

Proof. Lemma 4 in [16] states that

$$
\mathcal{L}\left(r, \infty, \frac{f^{\prime}}{f}\right)=O(\log (T(r, f))+O(\log r), \quad r \rightarrow \infty,
$$


possibly outside a set $E$ of finite linear measure. Let us assume that the statement is true for an integer $k \geq 1$ outside a set $E_{1}$ of finite linear measure. We know that

$$
\frac{f^{(k+1)}}{f}=\frac{f^{(k+1)}}{f^{(k)}} \cdot \frac{f^{(k)}}{f}
$$

and

$$
\mathcal{L}\left(r, \infty, \frac{f^{(k+1)}}{f^{(k)}}\right)=O\left(\log \left(T\left(r, f^{(k)}\right)\right)+O(\log r), \quad r \rightarrow \infty, r \notin E_{2} .\right.
$$

Applying the properties of $\log ^{+}$and by assumption, we get

$$
\begin{aligned}
& \mathcal{L}\left(r, \infty, \frac{f^{(k+1)}}{f}\right) \leq \mathcal{L}\left(r, \infty, \frac{f^{(k+1)}}{f^{(k)}}\right)+\mathcal{L}\left(r, \infty, \frac{f^{(k)}}{f}\right) \\
& =O\left(\log \left(T\left(r, f^{(k)}\right)\right)+O\left(\log (T(r, f))+O(\log r), \quad r \rightarrow \infty, r \notin E_{1} \cup E_{2} .\right.\right.
\end{aligned}
$$

By a generalisation of the lemma on the logarithmic derivative (see: [14]), apart from the set $E_{3}$ of finite linear measure we have

$$
T\left(r, f^{(k)}\right) \leq(k+1) T(r, f)+O(\log (T(r, f))+O(\log r) .
$$

This way we obtain

$$
\mathcal{L}\left(r, \infty, \frac{f^{(k+1)}}{f^{(k)}}\right)=O(\log (T(r, f))+O(\log r), \quad r \rightarrow \infty, r \notin E,
$$

where $E=\bigcup_{i=1}^{3} E_{i}$ is of finite linear measure.

Applying Proposition 2.2, it is possible to prove the following analogue of Theorem 2.1.

Theorem 2.3. Let $f$ be a transcendental meromorphic solution of

$$
f^{n} P(z, f)=Q(z, f),
$$

where $n$ is a positive integer, $P(z, f), Q(z, f)$ are polynomials in $f$ and its derivatives with meromorphic coefficients $a_{\nu}, b_{\nu}$, respectively, which are small with respect to $f$ in the sense that

$$
\mathcal{L}\left(r, \infty, a_{\nu}\right)=S(r, f), \quad \mathcal{L}\left(r, \infty, b_{\nu}\right)=S(r, f) .
$$

If the total degree $d$ of $Q(z, f)$ as a polynomial in $f$ and its derivatives is $d \leq n$, then

$$
\mathcal{L}(r, \infty, P(z, f))=S(r, f) .
$$

Proof. In the proof we apply ideas from the proof of Lemma 2.4.2 in [14]. Let

$$
f^{n} P(z, f)=Q(z, f),
$$

where

$$
\begin{aligned}
& P(z, f)=\sum_{\nu=0}^{t} a_{\nu}(z) f^{l_{0}^{\nu}} \cdot\left(f^{\prime}\right)^{l_{1}^{\nu}} \cdot \ldots \cdot\left(f^{(k)}\right)^{l_{k}^{\nu}}, l_{i}^{\nu} \in \mathbf{N}_{0}(i=0, \ldots, k, \nu=0, \ldots, t), \\
& Q(z, f)=\sum_{\nu=0}^{s} b_{\nu}(z) f^{j_{0}^{\nu}} \cdot\left(f^{\prime}\right)^{j_{1}^{\nu}} \cdot \ldots \cdot\left(f^{(k)}\right)^{j_{k}^{\nu}}, j_{i}^{\nu} \in \mathbf{N}_{0}(i=0, \ldots, k, \nu=0, \ldots, s),
\end{aligned}
$$

$a_{\nu}(\nu=0, \ldots, t), b_{\nu}(\nu=0, \ldots, s)$ are small with respect to $f$ in the sense that $\mathcal{L}\left(r, \infty, a_{\nu}\right)=S(r, f), \mathcal{L}\left(r, \infty, b_{\nu}\right)=S(r, f)$, and

$$
j_{0}^{\nu}+j_{1}^{\nu}+\ldots+j_{k}^{\nu} \leq d \leq n \quad(\nu=0, \ldots, s) .
$$


First, we consider the case when $0<|f(z)| \leq 1$, so $\frac{1}{|f(z)|} \geq 1$ and there are no poles of $a_{\nu}(\nu=0, \ldots, t)$ on the circle of radius $|z|=r$. Then

$$
\begin{aligned}
|P(z, f)| & =\left|\sum_{\nu=0}^{t} a_{\nu}(z) f^{l_{0}^{\nu}} \cdot\left(f^{\prime}\right)^{l_{1}^{\nu}} \cdot \ldots \cdot\left(f^{(k)}\right)^{l_{k}^{\nu}}\right| \leq \sum_{\nu=0}^{t}\left|a_{\nu}(z)\right||f|^{\nu_{0}^{\nu}} \cdot\left|f^{\prime}\right|_{1}^{l_{1}^{\nu}} \cdot \ldots \cdot\left|f^{(k)}\right|_{k}^{l_{k}^{\nu}} \\
& \leq \sum_{\nu=0}^{t}\left|a_{\nu}(z)\right|\left|\frac{f^{\prime}}{f}\right|^{l_{1}^{\nu}} \cdot \ldots \cdot\left|\frac{f^{(k)}}{f}\right|^{l_{k}^{\nu}} .
\end{aligned}
$$

It follows that

$$
\begin{aligned}
\log ^{+}|P(z, f)| & \leq \sum_{\nu=0}^{t}\left(\log ^{+}\left|a_{\nu}(z)\right|+\log ^{+}\left|\frac{f^{\prime}}{f}\right|^{l_{1}^{\nu}}+\ldots+\log ^{+}\left|\frac{f^{(k)}}{f}\right|^{l_{k}^{\nu}}\right)+\log t \\
& \leq \sum_{\nu=0}^{t}\left(\mathcal{L}\left(r, \infty, a_{\nu}\right)+\sum_{i=1}^{k} l_{i}^{\nu} \mathcal{L}\left(r, \infty, \frac{f^{(i)}}{f}\right)\right)+\log t:=M_{1}(|z|) .
\end{aligned}
$$

Next, let $1 \leq|f(z)|<\infty$, so $\frac{1}{|f(z)|} \leq 1$ and assume that there are no poles of $b_{\nu}(\nu=0, \ldots, s)$ on the circle of radius $|z|=r$. From (2) we have

SO

$$
P(z, f)=\frac{1}{f^{n}} Q(z, f),
$$

$$
\begin{aligned}
|P(z, f)| & =\left|\frac{1}{f^{n}} \sum_{\nu=0}^{s} b_{\nu}(z) f^{j_{0}^{\nu}} \cdot\left(f^{\prime}\right)^{j_{1}^{\nu}} \cdot \ldots \cdot\left(f^{(k)}\right)^{j_{k}^{\nu}}\right| \\
& \leq \sum_{\nu=0}^{s}\left|b_{\nu}(z)\right|\left|\frac{f^{\prime}}{f}\right|^{j_{1}^{\nu}} \cdot \ldots \cdot\left|\frac{f^{(k)}}{f}\right|^{j_{k}^{\nu}} \cdot\left(\frac{1}{|f|}\right)^{n-\left(j_{0}^{\nu}+\ldots+j_{k}^{\nu}\right)} \\
& \leq \sum_{\nu=0}^{s}\left|b_{\nu}(z)\right|\left|\frac{f^{\prime}}{f}\right|^{j_{1}^{\nu}} \cdot \ldots \cdot\left|\frac{f^{(k)}}{f}\right|^{j_{k}^{\nu}} \cdot
\end{aligned}
$$

It follows that

$$
\log ^{+}|P(z, f)| \leq \sum_{\nu=0}^{s}\left(\mathcal{L}\left(r, \infty, b_{\nu}\right)+\sum_{i=1}^{k} j_{i}^{\nu} \mathcal{L}\left(r, \infty, \frac{f^{(i)}}{f}\right)\right)+\log s:=M_{2}(|z|) .
$$

This way, apart from the circles where $f$ has zeros or $f, a_{\nu}, b_{\nu}$ have poles, we have

$$
\mathcal{L}(r, \infty, P(z, f)) \leq \max \left(M_{1}(|z|), M_{2}(|z|)\right) \text {. }
$$

Since $\mathcal{L}\left(r, \infty, a_{\nu}\right)=S(r, f)(\nu=0, \ldots, t)$ and $\mathcal{L}\left(r, \infty, b_{\nu}\right)=S(r, f)(\nu=0, \ldots, s)$, applying Proposition 2.2 we get

$$
M_{j}(r)=S(r, f) \quad(j=1,2),
$$

which completes the proof.

Let us now recall a well-known result of A. Z. Mohon'ko and V. D. Mohon'ko.

Theorem 2.4. [17] Let

$$
P\left(z, f, f^{\prime}, \ldots, f^{(n)}\right)=0
$$

be an algebraic differential equation $\left(P\left(z, u_{0}, u_{1}, \ldots, u_{n}\right)\right.$ is a polynomial in all arguments) and let $f$ be its transcendental meromorphic solution. If a constant a does not solve the equation, then $m\left(r, \frac{1}{f-a}\right)=S(r, f)$ and $\delta(a, f)=0$.

Next we shall prove an analogue of Theorem 2.4 for $\mathcal{L}(r, a, f)$. 
Theorem 2.5. If $f$ is a transcendental meromorphic solution of equation (3) and a constant a does not solve this equation, then $\mathcal{L}(r, a, f)=S(r, f)$ and $\beta(a, f)=0$.

Proof. We prove the statement in a similar way as Proposition 9.2.3 in [14]. Let $f$ be a transcendental meromorphic solution of equation (3). Then, for any constant $a \in \mathbf{C}$ the function $F(z):=f(z)-a$ is also a transcendental solution of a similar algebraic equation $\bar{P}\left(z, f, f^{\prime}, \ldots, f^{(n)}\right)=0$. Thus, we shall consider $F$ and a constant $a=0$. Let us assume that $a=0$ does not solve $\bar{P}\left(z, f, f^{\prime}, \ldots, f^{(n)}\right)=0$. We put

$$
\bar{P}\left(z, f, f^{\prime}, \ldots, f^{(n)}\right)=D(z)+Q\left(z, f, f^{\prime}, \ldots, f^{(n)}\right),
$$

where

$$
\begin{gathered}
Q\left(z, f, f^{\prime}, \ldots, f^{(n)}\right)=\sum_{\nu=0}^{t} p_{\nu}(z) f^{l_{0}^{\nu}} \cdot\left(f^{\prime}\right)^{l_{1}^{\nu}} \cdot \ldots \cdot\left(f^{(n)}\right)^{l_{n}^{\nu}} \\
l_{i}^{\nu} \in \mathbf{N}_{0}(i=0, \ldots, n, \nu=0, \ldots, t), \quad l_{0}^{\nu}+\ldots+l_{t}^{\nu} \geq 1(\nu=0, \ldots, t)
\end{gathered}
$$

all $p_{\nu}(\nu=0, \ldots, t)$ are polynomials,

$$
D(z):=\bar{P}(z, 0,0, \ldots, 0)
$$

and, by our assumption, $D(z) \not \equiv 0$.

In case $|F(z)|>1$ we have $\left|\frac{1}{F(z)}\right|<1$, so $\log ^{+}\left|\frac{1}{F(z)}\right|=0$.

If $|F(z)| \leq 1$, on the other hand, we have

$$
\begin{aligned}
\left|\frac{1}{F} Q\left(z, F, F^{\prime}, \ldots, F^{(n)}\right)\right| & \leq \sum_{\nu=0}^{t}\left|\frac{1}{F} p_{\nu}(z) F^{l_{0}^{\nu}} \cdot\left(F^{\prime}\right)^{l_{1}^{\nu}} \cdot \ldots \cdot\left(F^{(n)}\right)^{l_{n}^{\nu}}\right| \\
& =\sum_{\nu=0}^{t}\left|p_{\nu}(z)\right| \cdot\left|\frac{F^{\prime}}{F}\right|^{l_{1}^{\nu}} \cdot \ldots \cdot\left|\frac{F^{(n)}}{F}\right|^{l_{n}^{\nu}} \cdot|F|^{l_{0}^{\nu}+\ldots+l_{n}^{\nu}-1} \\
& \leq \sum_{\nu=0}^{t}\left|p_{\nu}(z)\right| \cdot\left|\frac{F^{\prime}}{F}\right|^{l_{1}^{\nu}} \cdot \ldots \cdot\left|\frac{F^{(n)}}{F}\right|^{l_{n}^{\nu}} .
\end{aligned}
$$

In all cases, since by (4) we have $D(z)=-Q\left(z, f, f^{\prime}, \ldots, f^{(n)}\right)$,

$$
\begin{aligned}
\mathcal{L}\left(r, \infty, \frac{1}{F}\right) & =\mathcal{L}\left(r, \infty, \frac{D}{F} \cdot \frac{1}{D}\right) \leq \mathcal{L}\left(r, \infty, \frac{D}{F}\right)+\mathcal{L}\left(r, \infty, \frac{1}{D}\right) \\
& =\mathcal{L}\left(r, \infty, \frac{Q}{F}\right)+\mathcal{L}\left(r, \infty, \frac{1}{D}\right) .
\end{aligned}
$$

By inequality (5) and Proposition 2.2, apart from a set of finite linear measure, we have

$$
\mathcal{L}\left(r, \infty, \frac{Q}{F}\right)=O(\log T(r, F))+O(\log r)=O(\log T(r, f))+O(\log r) .
$$

Since $f$ is transcendental we get

$$
\mathcal{L}\left(r, \infty, \frac{1}{f-a}\right)=\mathcal{L}\left(r, \infty, \frac{1}{F}\right)=S(r, f) .
$$

Remark 2.6. Theorem 2.5 is a (weaker) version of a result proved by V. D. Mohon'ko in [18]. The original proof is based on a method introduced by Petrenko. Applying Proposition 2.2 it is also possible to prove that algebraic equations with coefficients not necessarily polynomial, but meromorphic and small with respect to $f$ in the same sense as in Theorem 2.3, also have a similar property. 


\section{Painlevé equations and value distribution theory}

The six Painlevé equations have many applications in modern mathematics and mathematical physics and a number of remarkable properties (e.g., Hamiltonian structure, Bäcklund transformations). Their solutions have no movable branch points (so the equations possess the Painlevé property). These equations can also be obtained by similarity reductions from certain integrable partial differential equations (e.g., KdV, mKdV and others) [1, 2, 8, 12]. There are six Painlevé equations. They appeared as a result of the classification of second order ordinary differential equations with the Painlevé property

$$
f^{\prime \prime}=F\left(z, f, f^{\prime}\right), \quad f=f(z), \quad{ }^{\prime}=d / d z,
$$

where $F$ is rational in $f$, algebraic in $f^{\prime}$ and analytic in $z$. As a result of the classification only six equations were irreducible. Others were either transformed to them or to linear equations or to the first order equations or were integrated by quadrature. New functions, the Painlevé transcendents, were thus found. Later on it was shown that they are meromorphic functions on the universal cover of $\mathbf{C}$ with certain fixed singular points removed for some equations (see [25] and the references therein).

The second and the fourth Painlevé equations are given by

$$
\begin{aligned}
& \left(P_{2}\right) f^{\prime \prime}=2 f^{3}+z f+\alpha, \\
& \left(P_{4}\right) f^{\prime \prime}=\frac{f^{\prime 2}}{2 f}+\frac{3 f^{3}}{2}+4 z f^{2}+2\left(z^{2}-\alpha\right) f+\frac{\beta}{f},
\end{aligned}
$$

where $\alpha, \beta$ are arbitrary complex parameters and $f=f(z)$. Their solutions are meromorphic functions in the sense that every local solution has a continuation to a function meromorphic in $\mathbf{C}$. For recent proofs see Hinkkanen and Laine in [10] for $P_{2}$ or Steinmetz in [29] for both equations. The solutions are also known to be of finite order $[26,27,30]$. The deficiencies and ramification indices of solutions have been estimated both in case of $P_{2}$ and $P_{4}$. The estimates of deficiencies for $P_{2}$ are due to Schubart and Schubart and Wittich. The estimates of the ramification indices are due to Kieling.

Theorem 3.1. $[23,24,13]$ Transcendental solutions of $P_{2}$ fulfill the conditions:

1. $m(r, f)=O(\log r)$ and $\delta(\infty, f)=0$;

2. if $\alpha \neq 0$, then, for every $a \in \mathbf{C}$, we have $m\left(r, \frac{1}{f-a}\right)=O(\log r)$ and $\delta(a, f)=0$;

3 . in the case of $\alpha=0$ for every $a \in \mathbf{C} \backslash\{0\}$ we have $m\left(r, \frac{1}{f-a}\right)=O(\log r)$ and $\delta(a, f)=0$, and for $a=0$ we have $m\left(r, \frac{1}{f}\right) \leq \frac{1}{2} T(r, f)+O(\log r)$ and $\delta(0, f) \leq \frac{1}{2}$.

4. for every $a \in \mathbf{C} \backslash\{0\}$ we have $N_{1}\left(r, \frac{1}{f-a}\right) \leq \frac{1}{4} T(r, f)+O(\log r)$ and $\vartheta(a, f) \leq \frac{1}{4}$;

5. if $\alpha \neq 0$, then $N_{1}\left(r, \frac{1}{f}\right) \leq \frac{1}{5} T(r, f)+O(\log r)$ and $\vartheta(0, f) \leq \frac{1}{5}$, and if $\alpha=0$, then $N_{1}\left(r, \frac{1}{f}\right)=0$ and $\vartheta(0, f)=0$;

6. $N_{1}(r, f)=0$ and $\vartheta(\infty, f)=0$.

Let us recall the estimates for transcendental solutions of $P_{4}$ which were originally given by Steinmetz.

Theorem 3.2. [28] Transcendental solutions of $P_{4}$ fulfill the conditions:

1. $m(r, f)=O(\log r)$ and $\delta(\infty, f)=0$;

2. if $\beta \neq 0$, then for $a \in \mathbf{C}$ we have $m\left(r, \frac{1}{f-a}\right)=O(\log r)$ and $\delta(a, f)=0$;

3. if $\beta=0$ and $a \neq 0$, then we have $m\left(r, \frac{1}{f-a}\right)=O(\log r)$ and $\delta(a, f)=0$; 
4. if $\beta=0$ and if $f$ does not satisfy the Riccati differential equation $f^{\prime}=$ $\pm\left(f^{2}+2 z f\right)$, then $m\left(r, \frac{1}{f}\right) \leq \frac{1}{2} T(r, f)+O(\log r)$ and $\delta(0, f) \leq \frac{1}{2}$;

5. for every $a \in \mathbf{C} \backslash\{0\}, N_{1}\left(r, \frac{1}{f-a}\right) \leq \frac{1}{4} T(r, f)+O(\log r)$ and $\vartheta(a, f) \leq \frac{1}{4}$;

6. if $\beta \neq 0$, then $N_{1}\left(r, \frac{1}{f}\right)=0$ and $\vartheta(0, f)=0$;

7. if $\beta=0$, then $N_{1}\left(r, \frac{1}{f}\right)=\frac{1}{2} T(r, f)+O(\log r)$ and $\vartheta(0, f)=\frac{1}{2}$;

8. $N_{1}(r, f)=0$ and $\vartheta(\infty, f)=0$.

We can formulate results similar to the estimates of defects, but concerning deviations of the transcendental meromorphic solutions of $P_{2}$ and $P_{4}$.

Theorem 3.3. Transcendental meromorphic solutions of $P_{2}$ and $P_{4}$ have the following properties.

1. For solutions of $P_{2}(\alpha)$ the equalities $\mathcal{L}(r, a, f)=S(r, f)$ and $\beta(a, f)=0$ hold for all $a \in \overline{\mathbf{C}} \backslash\{0\}$. If $\alpha \neq 0$ we also have $\mathcal{L}(r, 0, f)=S(r, f)$ and $\beta(0, f)=0$.

2. If $f$ is a solution of $P_{4}(\alpha, \beta)$, then the equalities $\mathcal{L}(r, a, f)=S(r, f)$ and $\beta(a, f)=0$ hold for all $a \in \overline{\mathbf{C}} \backslash\{0\}$. If $\beta \neq 0$, then we also have $\mathcal{L}(r, 0, f)=$ $S(r, f)$ and $\beta(0, f)=0$.

Proof. 1. We consider transcendental meromorphic solutions of the equation $P_{2}(\alpha)$ and we write the equation in the form

$$
f^{2} P(z, f)=Q(z, f),
$$

with $P(z, f)=f$ and $Q(z, f)=\frac{1}{2}\left(f^{\prime \prime}-z f-\alpha\right)$. By Theorem 2.3 we have

$$
\mathcal{L}(r, f)=\mathcal{L}(r, P(z, f))=S(r, f) .
$$

The assumption that a constant $a \in \mathbf{C}$ is a solution of $P_{2}$ leads to the equation

$$
a z+2 a^{3}+\alpha \equiv 0 .
$$

It follows that we have a constant solution $a=0$ of $P_{2}$ only when $\alpha=0$. Applying Theorem 2.5 we may conclude that if $\alpha \neq 0$ we have $\mathcal{L}(r, a, f)=S(r, f)$ for all $a \in \overline{\mathbf{C}}$, and if $\alpha=0$ we have $\mathcal{L}(r, a, f)=S(r, f)$ for $a \in \overline{\mathbf{C}} \backslash\{0\}$.

2. We consider transcendental meromorphic solutions of $P_{4}(\alpha, \beta)(\alpha, \beta \in \mathbf{C})$ and write the equation in the form

$$
f^{3} P(z, f)=Q(z, f),
$$

with $P(z, f)=f$ and $Q(z, f)=\frac{1}{3}\left(2 f f^{\prime \prime}-\left(f^{\prime}\right)^{2}-8 z f^{3}-4\left(z^{2}-\alpha\right) f^{2}-2 \beta\right)$. By Theorem 2.3 we have

$$
\mathcal{L}(r, f)=\mathcal{L}(r, P(z, f))=S(r, f) .
$$

If we assume that a constant $a \in \mathbf{C}$ solves the equation $P_{4}(\alpha, \beta)$ we get the equality

$$
4 a^{2} z^{2}+8 a^{3} z+3 a^{4}-4 \alpha a^{2}+2 \beta \equiv 0 .
$$

If $\beta \neq 0$, then this equality does not hold. If $\beta=0$, then the only solution is $a=0$. By Theorem 2.5 and our previous considerations, if $\beta \neq 0$ we have $\mathcal{L}(r, a, f)=S(r, f)$ for all $a \in \overline{\mathbf{C}}$, and if $\beta=0$ we have $\mathcal{L}(r, a, f)=S(r, f)$ for $a \in \overline{\mathbf{C}} \backslash\{0\}$.

In this paper we are particularly interested in the so-called unified equation of $P_{4}$ and $P_{34}$. Equation $P_{34}$, also called equation XXXIV in [11, Ch. 14], is the second order equation of the form

$$
f^{\prime \prime}=\frac{\left(f^{\prime}\right)^{2}}{2 f}+B f(2 f-z)-\frac{A}{2 f}
$$


where $A$ and $B$ are fixed complex parameters. It follows from the relationship with $P_{2}$ that the solutions of $P_{34}$ are meromorphic (see the Appendix).

The unified equation of $P_{34}$ and $P_{4}$ was introduced in [20]. This equation has the following form:

$$
f^{\prime \prime}=\frac{\left(f^{\prime}\right)^{2}}{2 f}-\frac{\alpha}{2 f}+\beta f(2 f+z)+\gamma f(f+z)(3 f+z) .
$$

We shall use the notation $P_{4,34}$ (or $P_{4,34}(\alpha, \beta, \gamma)$ to underline the dependence on the parameters) for this equation. Equation $P_{4,34}$ admits the following scaling transformation: if $f(z)$ is a solution of $P_{4,34}(\alpha, \beta, \gamma)$, then $f(c z) / c$ is a solution of $P_{4,34}\left(\alpha, c^{3} \beta, c^{4} \gamma\right)$ [20]. It is shown in [20] that if $\beta=0, \gamma=0$, then equation (7) can easily be integrated with polynomial solutions

$$
f(z)=\frac{\left(C_{1}^{2}-\alpha\right) z^{2}}{4 C_{2}}+C_{1} z+C_{2} .
$$

In the following, we shall not consider the case $\beta=\gamma=0$.

If $\gamma=0, \beta \neq 0$, then, by changing the independent variable $t \rightarrow-t$ in (7) one obtains (6) with parameters $A=\alpha$ and $B=\beta$. If $\gamma \neq 0, \alpha=-\beta / 2, \beta=b^{3}, \gamma=2 d^{4}$ in (7), then $2 d f(t)$, where $t=\left(z+b^{3} /\left(4 d^{4}\right)\right) d$ is a solution of $P_{4}(\tilde{\alpha}, \tilde{\beta})$ with parameters $\tilde{\alpha}=b^{6} d^{-6} / 16=\beta^{2} /\left(4 \sqrt{2} \gamma^{3 / 2}\right)$ and $\tilde{\beta}=\beta[20]$. The properties of $P_{4,34}$ and $P_{34}$ not connected directly to the Nevanlinna theory will be discussed in the Appendix.

In the following we shall present some properties of the solutions of $P_{4,34}$ in two cases:

(C1) $\gamma=0, \beta \neq 0$

(C2) $\gamma \neq 0$.

Equation $P_{4,34}$ admits singular values 0 and $\infty$. Let us consider $\infty$ as the singular value of $f$, i.e., consider expansions of solutions around a movable pole $z_{0}$.

Theorem 3.4. The equation $P_{4,34}$ has the following polar behavior.

1. If $\gamma=0$, then an arbitrary solution of $P_{4,34}(\alpha, \beta, 0)$ has double poles. Moreover, equation $P_{4,34}(\alpha, \beta, 0)$ can be re-written in the form of a regular system at a pole $z=z_{0}$ for the variables $u(z)^{2}=1 / f(z)$ and $v(z)$ defined by

$$
f^{\prime}(z)=-1-\frac{\sqrt{2 \beta}}{u(z)^{3}}-\frac{\sqrt{\beta} z}{\sqrt{2} u(z)}-\frac{u(z)\left(\sqrt{2} \beta z^{2}-120 \sqrt{2} v(z)\right)}{24 \sqrt{\beta}}
$$

such that the functions $u(z)$ and $v(z)$ are analytic in the neighborhood of $z=z_{0}$ and $u\left(z_{0}\right)=0$ and $v\left(z_{0}\right)=a_{2}$, where $a_{2}$ is arbitrary.

2. If $\gamma \neq 0$, then an arbitrary solution of $P_{4,34}(\alpha, \beta, \gamma)$ has simple poles. Moreover, equation $P_{4,34}(\alpha, \beta, \gamma)$ can be re-written in the form of a regular system at a pole $z=z_{0}$ for the variables $u(z)=1 / f(z)$ and $v(z)$ defined by

$$
f^{\prime}(z)=-\frac{\sqrt{2 \gamma}}{\varepsilon u(z)^{2}}-\frac{\beta+2 z \gamma}{\sqrt{2 \gamma \varepsilon u(z)}}+\frac{\sqrt{2} \beta^{2}-8 \varepsilon \sqrt{\gamma} \gamma}{8 \varepsilon \sqrt{\gamma} \gamma}+\frac{(8 \sqrt{2 \gamma} v(z)-\varepsilon \beta-2 z \varepsilon \gamma) u(z)}{4 \varepsilon \gamma}
$$

such that the functions $u(z)$ and $v(z)$ are analytic in the neighborhood of $z=z_{0}$ and $u\left(z_{0}\right)=0$ and $v\left(z_{0}\right)=a_{2}$, where $a_{2}$ is arbitrary.

Proof. Case $(\mathrm{C} 1)$. Around a pole $z=z_{0}$ we have the following Laurent series expansion with $\xi=z-z_{0}$ :

$$
f(z)=\frac{2}{\beta \xi^{2}}-\frac{z_{0}}{3}-\frac{\xi}{2}+a_{2} \xi^{2}+\frac{\beta z_{0} \xi^{3}}{18}+\ldots
$$


where $a_{2}$ is arbitrary. Then it can be shown that, fixing one of the two branches in the definition of $u$, the system is given by

$$
\begin{aligned}
48 \sqrt{\beta} u^{\prime}(z)=24 & \sqrt{2} \beta+12 \sqrt{2} \beta z u(z)^{2}+24 \sqrt{\beta} u(z)^{3}+\sqrt{2} \beta z^{2} u(z)^{4}-120 \sqrt{2} u(z)^{4} v(z), \\
1440 \sqrt{2 \beta} v^{\prime}(z)= & 96 \sqrt{2 \beta} \beta z+18 \sqrt{2 \beta} u(z)^{2}\left(\beta z^{2}-120 v(z)\right)+\left(\beta z^{2}-120 v(z)\right)^{2} u(z)^{3} \\
& +12 \beta u(z)\left(12-12 \alpha+\beta z^{3}-120 z v(z)\right) .
\end{aligned}
$$

Computing the expansions of $u$ and $v$ using (8) we show that $u\left(z_{0}\right)=0$ and $v\left(z_{0}\right)=a_{2}$.

Case $(\mathrm{C} 2)$. Let $\gamma \neq 0$ and $f(z)$ be an arbitrary solution of $P_{4,34}(\alpha, \beta, \gamma)$. Then around a pole $z=z_{0}$ we have the following Laurent series expansion with $\xi=z-z_{0}$ and $\varepsilon^{2}=1$ :

(9) $f(z)=\frac{\varepsilon}{\sqrt{2 \gamma} \xi}-\frac{\beta+2 z_{0} \gamma}{4 \gamma}+\frac{\left(4 z_{0}^{2} \gamma^{2} \varepsilon+4 z_{0} \beta \gamma \varepsilon+3 \beta^{2} \varepsilon-16 \sqrt{2 \gamma} \gamma\right) \xi}{24 \sqrt{2 \gamma} \gamma}+a_{2} \xi^{2}+\ldots$,

where $a_{2}$ is arbitrary. Since $z_{0}=z-\xi$, we have the following expansion for the function $u(z)=1 / f(z)$ :

$$
u(z)=\sqrt{2 \gamma} \varepsilon \xi+\frac{(\beta+2 z \gamma) \xi^{2}}{2}+\frac{\sqrt{\gamma}\left(2 \beta z+2 \gamma z^{2}+\varepsilon \sqrt{2 \gamma}\right) \xi^{3}}{3 \varepsilon \sqrt{2}}+\ldots
$$

Then

$$
\xi=\frac{\varepsilon u(z)}{\sqrt{2 \gamma}}-\frac{(\beta+2 z \gamma) \varepsilon u(z)^{2}}{4 \sqrt{2 \gamma} \gamma}+\frac{\left(3 \beta^{2}+8 z \beta \gamma+8 z^{2} \gamma^{2}-2 \varepsilon \sqrt{2 \gamma} \gamma\right) \varepsilon u(z)^{3}}{24 \sqrt{2 \gamma} \gamma^{2}}+\ldots
$$

Substituting this into the expansion for $f^{\prime}(z)$ yields

$$
f^{\prime}(z)=-\frac{\sqrt{2 \gamma}}{\varepsilon u(z)^{2}}-\frac{\beta+2 z \gamma}{\sqrt{2 \gamma} \varepsilon u(z)}+\frac{\sqrt{2} \beta^{2}-8 \varepsilon \sqrt{\gamma} \gamma}{8 \varepsilon \sqrt{\gamma} \gamma}+\frac{\left(8 \sqrt{2 \gamma} a_{2}-\varepsilon \beta-2 z \varepsilon \gamma\right) u(z)}{4 \varepsilon \gamma}+\ldots
$$

Thus, introducing the function $v(z)$ as in the theorem in the second case, we get a regular system

$$
\begin{aligned}
8 \sqrt{\gamma} \gamma \varepsilon u^{\prime}(z)= & 8 \sqrt{2} \gamma^{2}+4 \sqrt{2} \gamma(\beta+2 \gamma z) u(z)+\left(8 \varepsilon \sqrt{\gamma} \gamma-\sqrt{2} \beta^{2}\right) u(z)^{2} \\
& +2 u(z)^{3}(\sqrt{\gamma}(\beta+2 \gamma z) \varepsilon-8 \sqrt{2} \gamma v(z)), \\
128 \varepsilon \sqrt{2 \gamma} \gamma^{2} v^{\prime}(z)= & \beta^{4}+64 \gamma^{3}-32 \alpha \gamma^{3}+32 \varepsilon \sqrt{2 \gamma} \gamma^{2} \beta z+32 \varepsilon \sqrt{2 \gamma} \gamma^{3} z^{2} \\
& -128 \gamma^{2}(\beta+2 \gamma z) v(z)--4 u(z)\left(\sqrt{\gamma}\left(\sqrt{2} \beta^{2}-8 \varepsilon \sqrt{\gamma} \gamma\right) \varepsilon(\beta+2 \gamma z)\right. \\
& \left.-16 \gamma\left(\beta^{2}-4 \varepsilon \sqrt{2 \gamma} \gamma\right) v(z)\right)++6 \gamma u(z)^{2}\left((\beta+2 \gamma z)^{2}\right. \\
& \left.-16 \varepsilon \sqrt{2 \gamma}(\beta+2 \gamma z) v(z)+128 \gamma v(z)^{2}\right) .
\end{aligned}
$$

Thus we can easily check the analyticity of $u$ and $v$ and by direct computation using (9) we can show that $u\left(z_{0}\right)=0$ and $v\left(z_{0}\right)=a_{2}$.

Next consider 0 as the singular value of $f$. This case is even simpler and we shall only present expansions and regular systems for the appropriately chosen functions $u(z)$ and $v(z)$.

Case 1. Assume $\alpha \neq 0$. Around a zero $z=z_{0}$ we have

$$
f(z)=\varepsilon \sqrt{\alpha}\left(z-z_{0}\right)+a_{2}\left(z-z_{0}\right)^{2}+\ldots,
$$


where $a_{2}$ is an arbitrary constant. We have $f(z)=u(z), f^{\prime}(z)=\varepsilon \sqrt{\alpha}(1+u(z) v(z))$, $\varepsilon^{2}=1$, and

$$
\begin{aligned}
u^{\prime}(z) & =\varepsilon \sqrt{\alpha}(1+u(z) v(z)), \\
2 \varepsilon \sqrt{\alpha} v^{\prime}(z) & =2 z(\beta+\gamma z)+4(\beta+2 \gamma z) u(z)+6 \gamma u(z)^{2}-\alpha v(z)^{2} .
\end{aligned}
$$

Note that $v\left(z_{0}\right)=2 a_{2} / \alpha$.

Case 2. If $\alpha=0$, then $f(z)=u(z)^{2}, f^{\prime}(z)=2 u(z) v(z)$ and, hence,

$$
\begin{aligned}
u^{\prime}(z) & =v(z), \\
2 v^{\prime}(z) & =z(\beta+\gamma z) u(z)+2(\beta+2 \gamma z) u(z)^{3}+3 \gamma u(z)^{5} .
\end{aligned}
$$

We also have $v\left(z_{0}\right)=\sqrt{a_{2}}$.

We remark that the regular systems written above can be used not only for proving convergence of expansions, but also for the proof of the Painlevé property (see, for instance, [25]). The computational part of the proof consists of finding the Laurent series expansion around a movable zero or a pole of an arbitrary solution, constructing a regular system for two auxiliary functions using this expansion and finding the so-called Lyapunov function with certain properties. The remaining details can be found in [25]. We shall present some more properties of equation $P_{4,34}$ in the Appendix.

Let us now formulate the main results concerning the distribution of $a$-points $(a \in \overline{\mathbf{C}})$ of a transcendental solution of $(7)$.

Theorem 3.5. Transcendental meromorphic solutions of $P_{4,34}(\alpha, \beta, \gamma)$ satisfy the conditions

1. $m(r, f)=S(r, f)$;

2. $m\left(r, \frac{1}{f-a}\right)=S(r, f)$ for all $a \in \mathbf{C} \backslash\{0\}$;

3. if $\alpha \neq 0$, then $m\left(r, \frac{1}{f}\right)=S(r, f)$;

4. if $\alpha=0$ and $\gamma \neq 0$, then $m\left(r, \frac{1}{f}\right) \leq \frac{1}{2} T(r, f)+S(r, f)$ unless $f$ fulfills the Riccati differential equation

$$
f^{\prime}=\varepsilon \sqrt{2 \gamma} f(f+z+\beta /(2 \gamma)) \quad \text { with } \quad \beta^{2}+4 \varepsilon \gamma \sqrt{2 \gamma}=0 \quad\left(\varepsilon^{2}=1\right),
$$

in which case $m\left(r, \frac{1}{f}\right) \leq T(r, f)+O(1)$;

5. if $\alpha=0$ and $\gamma=0$, then $m\left(r, \frac{1}{f}\right) \leq \frac{1}{2} T(r, f)+S(r, f)$.

As a consequence of the Clunie lemma we obtain point 1 of Theorem 3.5 and the following conclusion. tions.

Corollary 3.6. The equation $P_{4,34}$ does not admit transcendental entire solu-

Corollary 3.7. If $f$ is a transcendental meromorphic solution of $P_{4,34}(\alpha, \beta, \gamma)$ with $\alpha \neq 0$, then both in case (C1) and (C2) for all $a \in \overline{\mathbf{C}}$ we have

$$
\delta(a, f)=0,
$$

so the set $E_{N}(f)$ of Nevanlinna's defective values of $f$ is empty. For $P_{4,34}(0, \beta, \gamma)$, both in case $(C 1)$ and $(C 2)$, we have $E_{N}(f) \subseteq\{0\}$. Moreover, $\delta(0, f) \leq 1 / 2$, unless in case (C2) $f$ fulfills (11) and then $\delta(0, f)=1$.

Corollary 3.8. For a transcendental meromorphic solution $f$ of $P_{34}(A, B)$, we have $E_{N}(f)=\emptyset$ if $A \neq 0$ and $E_{N}(f) \subseteq\{0\}$ with $\delta(0, f) \leq 1 / 2$ if $A=0$. 
We should notice here that Theorem 3.5 agrees with the estimates from Theorem 3.2 for transcendental solutions of $P_{4}$ with an appropriate choice of parameters.

The following result gives estimates for deviations of solutions of $P_{4,34}$.

Theorem 3.9. Transcendental meromorphic solutions of $P_{4,34}$ satisfy the conditions

1. $\mathcal{L}(r, \infty, f)=S(r, f)$,

2. $\mathcal{L}(r, a, f)=S(r, f)$ for all $a \in \mathbf{C} \backslash\{0\}$.

If $\alpha \neq 0$ we also have $\mathcal{L}(r, 0, f)=S(r, f)$.

Corollary 3.10. If $f$ is a transcendental meromorphic solution of $P_{4,34}$, then for all $a \in \overline{\mathbf{C}} \backslash\{0\}$

$$
\beta(a, f)=0 .
$$

If $\alpha \neq 0$ also $\beta(0, f)=0$, so in this case the set $E_{\Pi}(f)$ of Petrenko's exceptional values of $f$ is empty.

In a particular case of $P_{34}$ we can also arrive at the following conclusions.

Corollary 3.11. A transcendental meromorphic solution $f$ of the equation $P_{34}(A, B)$ does not possess exceptional values in the sense of Petrenko if $A \neq 0$. If $A=0$ then $E_{\Pi}(f) \subseteq\{0\}$.

The following result describes multiplicity of $a$-points of a solution of (7) depending on the parameters $\alpha, \beta, \gamma$.

Theorem 3.12. Let $f$ be a transcendental solution of $P_{4,34}$.

1. For $P_{4,34}$ in case $(C 1)$, all the poles of $f$ are double and $\vartheta(\infty, f)=1 / 2$. For $P_{4,34}(\alpha, \beta, \gamma)$ in case $(C 2)$ all the poles of $f$ are simple and $\vartheta(\infty, f)=0$.

2. For $P_{4,34}(\alpha, \beta, \gamma),(\alpha \neq 0)$ all the zeros of $f$ are simple and $\vartheta(0, f)=0$. For $P_{4,34}(0, \beta, \gamma)$, the zeros of non-zero solutions are double. Thus we have $\vartheta(0, f) \leq \frac{1}{2}$ in case $(C 1)$ and in case (C2) unless $f$ fulfills the equation (11), which then means that $\vartheta(0, f)=0$.

3. For $a \neq 0$, we have $\vartheta(a, f) \leq \frac{1}{4}$.

The following conclusion concerning solutions of $P_{34}$ follows from Theorem 3.12.

Corollary 3.13. A transcendental meromorphic solution $f$ of $P_{34}$ satisfies the conditions:

1. all the poles of $f$ are double and $\vartheta(\infty, f)=1 / 2$;

2. for $P_{34}(A, B),(A \neq 0)$ all the zeros of $f$ are simple and $\vartheta(0, f)=0$, for $P_{34}(0, B)$, the zeros are double and $\vartheta(0, f) \leq \frac{1}{2}$;

3. if $a \in \mathbf{C} \backslash\{0\}$, we have $\vartheta(a, f) \leq \frac{1}{4}$.

Moreover, Theorem 3.12 agrees with the estimates in Theorem 3.2 for an appropriate choice of parameters.

\section{Proof of Theorem 3.5}

1. Let $f$ be a transcendental meromorphic solution of the equation (7). In case $(\mathrm{C} 2)$ we write the equation in the form

$$
f^{3} P(z, f)=Q(z, f),
$$

where

$$
P(z, f)=f, Q(z, f)=\frac{1}{3 \gamma} f f^{\prime \prime}-\frac{1}{6 \gamma}\left(f^{\prime}\right)^{2}-\left(\frac{4}{3} z+\frac{2 \beta}{3 \gamma}\right) f^{3}-\left(\frac{1}{3} z^{2}+\frac{\beta}{3 \gamma} z\right) f^{2}+\frac{\alpha}{6 \gamma} .
$$


As we can see both $P$ and $Q$ have constant or polynomial coefficients which are small comparing to $f$, and the degree of $Q$ with respect to $f$ and its derivatives equals 3 . In case $(\mathrm{C} 1)$ we put $(7)$ in the form

$$
f^{2} P(z, f)=Q(z, f)
$$

with

$$
P(z, f)=f, \quad Q(z, f)=\frac{1}{2 \beta} f f^{\prime \prime}-\frac{1}{4 \beta}\left(f^{\prime}\right)^{2}-\frac{1}{2} z f^{2}+\frac{\alpha}{4 \beta}
$$

and $\operatorname{deg} Q=2$. Thus the conditions of Theorem 2.1 are fulfilled in both cases, so we obtain

$$
m(r, f)=m(r, P(z, f))=S(r, f) .
$$

2. and 3. Assuming that a constant $a \in \mathbf{C}$ is a solution of (7) we obtain the equation

$$
2 \gamma a^{2} z^{2}+\left(2 \beta a^{2}+8 \gamma a^{3}\right) z+6 \gamma a^{4}+4 \beta a^{3}-\alpha \equiv 0 .
$$

This equation has solutions only in two cases:

Case $1 .(\alpha, \beta, \gamma)=(0,0,0)$. This is the case which we leave out of our considerations.

Case 2. $\alpha=0,(\beta, \gamma) \neq(0,0)$. Then the only solution is $a=0$.

This way, if we assume that $\alpha \neq 0$ then in cases (C1) and (C2) no constant solves (7) so then by Theorem 2.4 for any $a \in \mathbf{C}$ we have

$$
m\left(r, \frac{1}{f-a}\right)=S(r, f)
$$

4. Let us now estimate $m\left(r, \frac{1}{f}\right)$ when $f$ is a solution of (7) with $\alpha=0$ in case (C2). We follow the ideas from [28]. Consider an auxiliary function

$$
F(z):=\frac{\left(f^{\prime}(z)\right)^{2}}{2 f(z)}-\gamma(f(z))^{3}-(2 \gamma z+\beta)(f(z))^{2}-\left(\gamma z^{2}+\beta z\right) f(z) .
$$

Differentiating and applying (7) we get

$$
F^{\prime}(z)=-2 \gamma f^{2}(z)-(2 \gamma z+\beta) f(z) .
$$

As in case (C2) the poles of $f$ are simple (see the expansion (9)), it follows that the poles of $F$ are also simple. In fact, the expansion of $F$ around a pole $z_{0}$ is of the form

$$
F(z)=\frac{1}{z-z_{0}}+\sum_{n=0}^{\infty} a_{n}\left(z-z_{0}\right)^{n}, \quad a_{n} \in \mathbf{C} .
$$

Moreover, the poles of $F$ appear only at the poles of $f$.

We put $\Xi(z):=\frac{F(z)}{f(z)}$. Then

$$
\frac{f^{\prime}(z)}{f(z)} \Xi(z)+\Xi^{\prime}(z)=\frac{F^{\prime}(z)}{f(z)}=-2 \gamma f(z)-2 \gamma z-\beta .
$$

The function $\Xi$ is analytic at the poles of $f$ and, again by the expansion (9), if $z_{0}$ is a pole of $f$ then $\Xi\left(z_{0}\right)=\varepsilon \sqrt{2 \gamma}\left(\varepsilon^{2}=1\right)$. Assume that $\Xi(z) \not \equiv \varepsilon \sqrt{2 \gamma}$, so Nevanlinna theory is applicable to $\Xi$. We have shown that $m(r, f)=S(r, f)$, so from 
the properties of $\log ^{+}$and from the lemma on logarithmic derivative we can estimate $m(r, \Xi)$ :

$$
\begin{aligned}
m(r, \Xi) & =m\left(r, \frac{1}{2}\left(\frac{f^{\prime}}{f}\right)^{2}-\gamma f^{2}-(2 \gamma z+\beta) f-\left(\gamma z^{2}+\beta z\right)\right) \\
& \leq 2 m\left(r, \frac{f^{\prime}}{f}\right)+3 m(r, f)+O(\log r)=S(r, f) .
\end{aligned}
$$

Since at every pole $z_{0}$ of $f$ we have $\Xi^{2}\left(z_{0}\right)=2 \gamma$, by the first main theorem of Nevanlinna we get

$$
\begin{aligned}
N(r, f) & \leq N\left(r, \frac{1}{\Xi^{2}-2 \gamma}\right) \leq T\left(r, \frac{1}{\Xi^{2}-2 \gamma}\right) \\
& \leq 2 T(r, \Xi)+O(1)=2 N(r, \Xi)+S(r, f) .
\end{aligned}
$$

Poles of $\Xi$ may appear only at the zeros of $f$ as $\Xi$ is analytic at the poles of $f$. As both the poles of $\Xi$ and the zeros of $f$ are double, by the first main theorem of Nevanlinna,

$$
N(r, \Xi) \leq N\left(r, \frac{1}{f}\right)=T(r, f)-m\left(r, \frac{1}{f}\right)+O(1) .
$$

Applying (14) and (13) we obtain

$m\left(r, \frac{1}{f}\right) \leq T(r, f)-N(r, \Xi)+O(1) \leq T(r, f)-\frac{1}{2} N(r, f)+S(r, f) \leq \frac{1}{2} T(r, f)+S(r, f)$.

Assume now that $\Xi(z) \equiv \varepsilon \sqrt{2 \gamma}$. Then, by (12) a solution of (7) fulfills the Riccati differential equation

$$
f^{\prime}=\varepsilon \sqrt{2 \gamma} f(f+z+\beta /(2 \gamma)) \quad \text { with } \quad \beta^{2}+4 \varepsilon \gamma \sqrt{2 \gamma}=0 \quad\left(\varepsilon^{2}=1\right) .
$$

In this case for a non-zero solution we have $f(z) \neq 0$, so $N\left(r, \frac{1}{f}\right)=0$ (see Theorem 9.1 .12 in [14]). The conclusion then follows by applying the first main theorem of Nevanlinna.

5. Let now $f$ be a transcendental solution of (7) with $\alpha=0$ in case (C1). Then $P_{4,34}$ has the form

$$
f^{\prime \prime}=\frac{\left(f^{\prime}\right)^{2}}{2 f}+2 \beta f^{2}+\beta z f
$$

As in point 4 , we consider an auxiliary function

$$
F(z):=\frac{\left(f^{\prime}(z)\right)^{2}}{2 f(z)}-\beta(f(z))^{2}-\beta z f(z) .
$$

Differentiating and applying (7) we get

$$
F^{\prime}(z)=-\beta f(z)
$$

From (8) we know that all the poles of $f$ are double, so all the poles of $F^{\prime}$ are also double. We put $\Xi(z):=\frac{F(z)}{f(z)}$. Then

$$
\frac{f^{\prime}(z)}{f(z)} \Xi(z)+\Xi^{\prime}(z)=\frac{F^{\prime}(z)}{f(z)}=-\beta .
$$


Thus $\Xi$ is analytic at each pole $z_{0}$ of $f$, with the expansion

$$
\Xi(z)=\beta\left(z-z_{0}\right)+\sum_{n=2}^{\infty} a_{n}\left(z-z_{0}\right)^{n}, \quad a_{n} \in \mathbf{C} .
$$

Notice that $\Xi(z) \not \equiv 0$. The only singularities of $\Xi$ are poles, which appear at zeros of $f$ with the expansion

$$
\Xi(z)=\frac{2}{\left(z-z_{0}\right)^{2}}+\sum_{n=-1}^{\infty} a_{n}\left(z-z_{0}\right)^{n}, \quad a_{n} \in \mathbf{C}
$$

for a zero $z_{0}$ of $f$. As $m(r, f)=S(r, f)$, applying properties of $\log ^{+}$and the lemma on logarithmic derivative we get

$$
m(r, \Xi)=m\left(r, \frac{1}{2}\left(\frac{f^{\prime}}{f}\right)^{2}-\beta f-\beta z\right) \leq 2 m\left(r, \frac{f^{\prime}}{f}\right)+m(r, f)+O(\log r)=S(r, f) .
$$

Then, as the zeros of $\Xi$ at the poles of $f$ are simple,

$$
\frac{1}{2} N(r, f) \leq N\left(r, \frac{1}{\Xi}\right) \leq T(r, \Xi)+O(1)=N(r, \Xi)+S(r, f) .
$$

Poles of $\Xi$ appear only at the zeros of $f$, so by the first main theorem of Nevanlinna,

$$
N(r, \Xi) \leq N\left(r, \frac{1}{f}\right)=T(r, f)-m\left(r, \frac{1}{f}\right)+O(1) .
$$

It follows from (16) and then (15) that

$$
\begin{aligned}
m\left(r, \frac{1}{f}\right) & \leq T(r, f)-N(r, \Xi)+O(1) \leq T(r, f)-\frac{1}{2} N(r, f)+S(r, f) \\
& =m(r, f)+\frac{1}{2} N(r, f)+S(r, f) \leq \frac{1}{2} T(r, f)+S(r, f) .
\end{aligned}
$$

\section{Proof of Theorem 3.9}

1. Let $f$ be a transcendental meromorphic solution of the equation (7). We proceed as in point 1 of Theorem 3.5. In case $(\mathrm{C} 2)$ we write the equation in the form $f^{3} P(z, f)=Q(z, f)$ with

$P(z, f)=f, Q(z, f)=\frac{1}{3 \gamma} f f^{\prime \prime}-\frac{1}{6 \gamma}\left(f^{\prime}\right)^{2}-\left(\frac{4}{3} z+\frac{2 \beta}{3 \gamma}\right) f^{3}-\left(\frac{1}{3} z^{2}+\frac{\beta}{3 \gamma} z\right) f^{2}+\frac{\alpha}{6 \gamma}$.

This way $\operatorname{deg} Q=3$ with respect to $f$ and its derivatives. Both $P$ and $Q$ have constant or polynomial coefficients, which are small with respect to $f$. By Theorem 2.3, we obtain $\mathcal{L}(r, f)=S(r, f)$. In case $(\mathrm{C} 1)$ we put $(7)$ in the form

$$
f^{2} P(z, f)=Q(z, f),
$$

with

$$
P(z, f)=f, \quad Q(z, f)=\frac{1}{2 \beta} f f^{\prime \prime}-\frac{1}{4 \beta}\left(f^{\prime}\right)^{2}-\frac{1}{2} z f^{2}+\frac{\alpha}{4 \beta},
$$

so we can see that $\operatorname{deg} Q=2 . f^{2} P(z, f)=Q(z, f)$ with $P(z, f)=f$ and $\operatorname{deg} Q=2$. Thus, again, the conditions of Theorem 2.3 are fulfilled, so we obtain

$$
\mathcal{L}(r, f)=\mathcal{L}(r, P(z, f))=S(r, f) .
$$


2. As we have already noticed in the proof of Theorem 3.5, if a constant $a \in \mathbf{C}$ were a solution of (7) we would obtain the equation

$$
2 \gamma a^{2} z^{2}+\left(2 \beta a^{2}+8 \gamma a^{3}\right) z+6 \gamma a^{4}+4 \beta a^{3}-\alpha \equiv 0 .
$$

Thus $a$ fulfills (7) only if $a=0$ for $\alpha=0$. If $\alpha \neq 0$ then no constant solves (7) so by Theorem 2.5, for any $a \in \mathbf{C}$ we have

$$
\mathcal{L}\left(r, \frac{1}{f-a}\right)=S(r, f) .
$$

\section{Proof of Theorem 3.12}

1. It follows from Theorem 3.5 that a transcendental solution of $P_{4,34}(\alpha, \beta, \gamma)$ has an infinite number of poles as $m(r, f)=S(r, f)$. For $P_{4,34}(\alpha, \beta, 0)$ we have

$$
\begin{aligned}
\vartheta(\infty, f) & =\liminf _{r \rightarrow \infty} \frac{N_{1}(r, f)}{T(r, f)}=\liminf _{r \rightarrow \infty} \frac{N(r, f)-\bar{N}(r, f)}{T(r, f)} \\
& =\liminf _{r \rightarrow \infty} \frac{\frac{1}{2} N(r, f)}{T(r, f)}=\liminf _{r \rightarrow \infty} \frac{\frac{1}{2} T(r, f)+S(r, f)}{T(r, f)}=\frac{1}{2}
\end{aligned}
$$

as, by (8), all the poles are double. In case (C2) each pole is simple, as the expansion (9) shows. Therefore the index of multiplicity of poles

$$
\vartheta(\infty, f)=\liminf _{r \rightarrow \infty} \frac{N_{1}(r, f)}{T(r, f)}=\liminf _{r \rightarrow \infty} \frac{N(r, f)-\bar{N}(r, f)}{T(r, f)}=0
$$

in this case.

2. Let $\alpha \neq 0$. It follows from (10) that if $f$ is a solution of $P_{4,34}$ then all the zeros of $f$ are simple. Thus $\bar{N}(r, 0, f)=N(r, 0, f)$ and $\vartheta(0, f)=0$.

For $P_{4,34}(0, \beta, \gamma)$ all the zeros of a non-zero solution are double. It follows from point 5 in Theorem 3.5 and by the first main theorem, that in case $(\mathrm{C} 1)$ we get

$$
\vartheta(0, f)=\liminf _{r \rightarrow \infty} \frac{N\left(r, \frac{1}{f}\right)-\bar{N}\left(r, \frac{1}{f}\right)}{T(r, f)}=\liminf _{r \rightarrow \infty} \frac{\frac{1}{2} N\left(r, \frac{1}{f}\right)}{T(r, f)} \leq \frac{1}{2} .
$$

In case (C2), by point 4 in Theorem 3.5 and by the first main theorem, we have

$$
N\left(r, \frac{1}{f}\right)-\bar{N}\left(r, \frac{1}{f}\right)=\frac{1}{2} N\left(r, \frac{1}{f}\right) \leq \frac{1}{2} T(r, f)+S(r, f),
$$

so $\vartheta(0, f) \leq \frac{1}{2}$, provided that $f$ does not fulfill the Riccati differential equation (11). If (11) holds, then $\vartheta(0, f)=0$.

3. We again follow the ideas from [28]. Let $f$ be a transcendental meromorphic solution of $P_{4,34}(\alpha, \beta, \gamma)$. If for a fixed $a \in \mathbf{C} \backslash\{0\}$ we assume that all but possibly a finite number of $a$-points are single, then we instantly get $\vartheta(a, f)=0$. If, on the other hand we assume that at a point $z_{0}$ the equalities $f\left(z_{0}\right)=a, f^{\prime}\left(z_{0}\right)=0$ and $f^{\prime \prime}\left(z_{0}\right)=0$ hold, we get

$$
2 \gamma a^{2} z_{0}^{2}+\left(8 \gamma a^{3}+2 \beta a^{2}\right) z_{0}+6 \gamma a^{4}+4 \beta a^{3}-\alpha=0 .
$$

Since $\beta, \gamma \neq 0$, it means that for each fixed value $a$ only at most two $a$-points may have multiplicity 3 or higher. We now put

$$
A=\left\{z \in \mathbf{C}: f(z)=a, f^{\prime}(z)=0\right\}
$$


and consider the set

$A^{*}:=\left\{z \in A: 2 \gamma a^{2} z^{2}+\left(8 \gamma a^{3}+2 \beta a^{2}\right) z+6 \gamma a^{4}+4 \beta a^{3}-\alpha \neq 0,2 \gamma(2 a+z)+\beta \neq 0\right\}$ of double $a$-points of $f$. We have the following expansion around an $a$-point $z_{0}$ :

$$
\begin{aligned}
f(z)= & a+\left(\frac{1}{2} a z_{0}^{2} \gamma+2 a^{2} z_{0} \gamma+\frac{3}{2} a^{3} \gamma+\frac{1}{2} a z_{0} \beta+a^{2} \beta-\frac{\alpha}{4 a}\right)\left(z-z_{0}\right)^{2} \\
& +\frac{1}{6}\left(a \beta+4 a^{2} \gamma+2 a z_{0} \gamma\right)\left(z-z_{0}\right)^{3}+\ldots
\end{aligned}
$$

Let us assume that $A^{*}$ is not finite, otherwise we trivially get $\vartheta(a, f)=0$ since then the whole set of multiple $a$-points would be finite. Similarly as in the proof of Theorem 3.5 we consider an auxiliary function

$$
F(z):=\frac{\left(f^{\prime}(z)\right)^{2}}{2 f(z)}-\frac{\alpha}{2 f(z)}-\gamma(f(z))^{3}-(2 \gamma z+\beta)(f(z))^{2}-\left(\gamma z^{2}+\beta z\right) f(z) .
$$

Then, after applying $P_{4,34}(\alpha, \beta, \gamma)$, we get

$$
F^{\prime}(z)=-2 \gamma f^{2}(z)-(2 \gamma z+\beta) f(z) .
$$

We introduce another auxiliary function

$$
\begin{aligned}
F_{a}(z):= & \frac{\left(f^{\prime}(z)\right)^{2}}{2 f(z)}-\frac{\alpha}{2}\left(\frac{1}{f(z)}-\frac{1}{a}\right)-\gamma\left((f(z))^{3}-a^{3}\right) \\
& -(2 \gamma z+\beta)\left((f(z))^{2}-a^{2}\right)-\left(\gamma z^{2}+\beta z\right)(f(z)-a) .
\end{aligned}
$$

Thus

$$
\begin{aligned}
F_{a}(z) & =F(z)+\frac{\alpha}{2 a}+\gamma a^{3}+(2 \gamma z+\beta) a^{2}+\left(\gamma z^{2}+\beta z\right) a, \\
F_{a}^{\prime}(z) & =F^{\prime}(z)+2 \gamma a^{2}+(2 \gamma z+\beta) a=-2 \gamma\left((f(z))^{2}-a^{2}\right)-(2 \gamma z+\beta)(f(z)-a), \\
F_{a}^{\prime \prime}(z) & =-4 \gamma f(z) f^{\prime}(z)-2 \gamma(f(z)-a)-(2 \gamma z+\beta) f^{\prime}(z), \\
F_{a}^{\prime \prime \prime}(z) & =-4 \gamma f^{\prime}(z)\left(f^{\prime}(z)+1\right)-f^{\prime \prime}(z)(4 \gamma f(z)+2 \gamma z+\beta) .
\end{aligned}
$$

For $\xi \in A^{*}$ we have

$$
F_{a}(\xi)=0, \quad F_{a}^{\prime}(\xi)=0, \quad F_{a}^{\prime \prime}(\xi)=0 \quad \text { and } \quad F_{a}^{\prime \prime \prime}(\xi) \neq 0
$$

so all the elements in $A^{*}$ are zeros of $F_{a}$ of multiplicity 3 . Consider

$$
\Xi_{a}(z):=\frac{F_{a}(z)}{f(z)-a} .
$$

Then each $\xi \in A^{*}$ is a simple zero of $\Xi_{a}$ and

$\Xi_{a}^{\prime}(z)(f(z)-a)+\Xi_{a}(z) f^{\prime}(z)=F_{a}^{\prime}(z)=-(f(z)-a)(\beta+2(a+z) \gamma+2 \gamma f(z))$.

Case (C1). The auxiliary functions take the form:

$$
F(z)=\frac{\left(f^{\prime}(z)\right)^{2}}{2 f(z)}-\frac{\alpha}{2 f(z)}-\beta(f(z))^{2}-\beta z f(z),
$$

So

$$
\begin{aligned}
F^{\prime}(z) & =-\beta f(z), \\
F_{a}(z) & =\frac{\left(f^{\prime}(z)\right)^{2}}{2 f(z)}-\frac{\alpha}{2}\left(\frac{1}{f(z)}-\frac{1}{a}\right)-\beta\left((f(z))^{2}-a^{2}\right)-\beta z(f(z)-a) \\
& =F(z)+\frac{\alpha}{2 a}+\beta a^{2}+\beta z a,
\end{aligned}
$$




$$
F_{a}^{\prime}(z)=F^{\prime}(z)+\beta a=-\beta(f(z)-a), \quad F_{a}^{\prime \prime}(z)=-\beta f^{\prime}(z) \quad \text { and } \quad F_{a}^{\prime \prime \prime}(z)=-\beta f^{\prime \prime}(z) .
$$

By (8), each pole $z_{0}$ of $f$ is double, so we conclude that $\Xi_{a}(z)$ is analytic at $z_{0}$, $\Xi_{a}\left(z_{0}\right)=0$ and the zero is simple. Thus the poles of $F_{a}$ may appear only at the poles of $f$. If $a$ is a multiple $a$-point of $f$ then, by (17) and properties of $F_{a}$, it is a zero of $\Xi_{a}$. Therefore the only singularities of $\Xi_{a}$ are poles, which may appear at simple $a$-points of $f$. Using (17) and the first main theorem,

$$
N\left(r, \Xi_{a}\right) \leq N(r, a, f)-2 N_{1}(r, a, f)+O(\log r) \leq T(r, f)-2 N_{1}(r, a, f)+O(\log r) .
$$

By the definition of $\Xi_{a}$,

$$
m\left(r, \Xi_{a}\right)=m\left(r, \frac{1}{2} \frac{\left(f^{\prime}\right)^{2}}{f(f-a)}+\frac{\alpha}{2 a} \frac{1}{f}-\beta(f+a)-\beta z\right) .
$$

Applying properties of $\log ^{+}$we get

$$
m\left(r, \Xi_{a}\right) \leq m\left(r, \frac{f^{\prime}}{f}\right)+m\left(r, \frac{f^{\prime}}{f-a}\right)+m\left(r, \frac{1}{f}\right)+m(r, f)+O(\log r)
$$

if $\alpha \neq 0$, and

$$
m\left(r, \Xi_{a}\right) \leq m\left(r, \frac{f^{\prime}}{f}\right)+m\left(r, \frac{f^{\prime}}{f-a}\right)+m(r, f)+O(\log r)
$$

if $\alpha=0$.

For $\alpha \neq 0$ we apply lemma on the logarithmic derivative, points 1 and 3 in Theorem 3.5, and for $\alpha=0$ we just apply lemma on the logarithmic derivative and point 1 in Theorem 3.5. In both cases we obtain

$$
m\left(r, \Xi_{a}\right)=S(r, f) .
$$

This way we have

$$
T\left(r, \Xi_{a}\right) \leq T(r, f)-2 N_{1}(r, a, f)+S(r, f) .
$$

Since $\Xi_{a} \not \equiv 0$,

$$
N(r, f) \leq N\left(r, \frac{1}{\Xi_{a}^{2}}\right) \leq 2 T\left(r, \Xi_{a}\right)+O(1) .
$$

Applying (19) and then (18), we have

$$
T(r, f) \leq 2 T\left(r, \Xi_{a}\right)+S(r, f) \leq 2 T(r, f)-4 N_{1}(r, a, f)+S(r, f),
$$

so finally,

$$
N_{1}(r, a, f) \leq \frac{1}{4} T(r, f)+S(r, f) .
$$

Case (C2). If $\gamma \neq 0$ then each pole $z_{0}$ of $f$ is simple. By the Laurent expansion (9), we conclude that $\Xi_{a}(z)$ is analytic at $z_{0}$ and $\Xi_{a}\left(z_{0}\right)=\varepsilon \sqrt{2 \gamma},(\varepsilon= \pm 1)$. If $\Xi_{a} \equiv \varepsilon \sqrt{2 \gamma}$, by similar considerations as in the proof of point 4 of Theorem 3.5 we obtain $N\left(r, \frac{1}{f-a}\right)=0$. Let then $\Xi_{a} \not \equiv \varepsilon \sqrt{2 \gamma}$. The poles of $F_{a}$ may appear only at the poles of $f$. Therefore, by the definition of $\Xi_{a}$, we can see that the only singularities of $\Xi_{a}$ are poles, which may appear at simple $a$-points of $f$. Applying (17) and the first main theorem,

$$
N\left(r, \Xi_{a}\right) \leq N(r, a, f)-2 N_{1}(r, a, f)+O(\log r) \leq T(r, f)-2 N_{1}(r, a, f)+O(\log r) .
$$


By definition of $\Xi_{a}$,

$$
\begin{gathered}
m\left(r, \Xi_{a}\right)=m\left(r, \frac{1}{2} \frac{\left(f^{\prime}\right)^{2}}{f(f-a)}+\frac{\alpha}{2 a} \frac{1}{f}-\gamma f^{2}-(2 \gamma z+\gamma a+\beta) f\right. \\
\left.-\gamma z^{2}-(\beta-2 \gamma a) z-\gamma a^{2}-a \beta\right) .
\end{gathered}
$$

Applying properties of $\log ^{+}$we get

$$
m\left(r, \Xi_{a}\right) \leq m\left(r, \frac{f^{\prime}}{f}\right)+m\left(r, \frac{f^{\prime}}{f-a}\right)+m\left(r, \frac{1}{f}\right)+3 m(r, f)+O(\log r)
$$

if $\alpha \neq 0$, and

$$
m\left(r, \Xi_{a}\right) \leq m\left(r, \frac{f^{\prime}}{f}\right)+m\left(r, \frac{f^{\prime}}{f-a}\right)+3 m(r, f)+O(\log r)
$$

if $\alpha=0$.

For $\alpha \neq 0$ we apply lemma on the logarithmic derivative, points 1 and 3 in Theorem 3.5, and for $\alpha=0$ we just apply lemma on the logarithmic derivative and point 1 in Theorem 3.5. In both cases we obtain

$$
m\left(r, \Xi_{a}\right)=S(r, f) .
$$

This way we have

$$
T\left(r, \Xi_{a}\right) \leq T(r, f)-2 N_{1}(r, a, f)+S(r, f) .
$$

Since $\Xi_{a} \not \equiv \varepsilon \sqrt{2 \gamma}$

$$
N(r, f) \leq N\left(r, \frac{1}{\Xi_{a}^{2}-2 \gamma}\right) \leq 2 T\left(r, \Xi_{a}\right)+O(1) .
$$

Applying (21) and then (20), we have

$$
T(r, f) \leq 2 T\left(r, \Xi_{a}\right)+S(r, f) \leq 2 T(r, f)-4 N_{1}(r, a, f)+S(r, f),
$$

so finally,

$$
N_{1}(r, a, f) \leq \frac{1}{4} T(r, f)+S(r, f)
$$

\section{Appendix}

Equation $\boldsymbol{P}_{\mathbf{3 4}}$. Equation (6), referred to as $P_{34}$ is related to the second Painlevé equation $P_{2}$ in the following way [4]. Equation $P_{2}$ admits a Hamiltonian formulation [21]. If

$$
H=1 / 2 y^{2}-\left(x^{2}+z / 2\right) y-(\alpha+1 / 2) x
$$

is a Hamiltonian of the system

$$
\frac{d x}{d z}=\frac{\partial H}{\partial y}, \quad \frac{d y}{d z}=-\frac{\partial H}{\partial x},
$$

then by eliminating the function $y=\left(z+2 x^{2}+2 x^{\prime}\right) / 2$, the function $x$ satisfies $P_{2}$. By eliminating $x=\left(2 y^{\prime}-2 \alpha-1\right) /(4 y)$ between these equations, it is easy to show that the function $y$ satisfies the equation (6) with $A=(2 \alpha+1)^{2} / 4, B=1$. Clearly, since solutions of $P_{2}$ are meromorphic in $\mathbf{C}$, then the solutions of $P_{34}(A, 1)$ are also meromorphic.

Equation $P_{34}$ has a scaling symmetry: if $f(z)$ is a solution of $P_{34}$ with the parameters $A, B=1$, then $B^{-1 / 3} f\left(B^{1 / 3} z\right)$ is a solution of $P_{34}$ with the parameters $A$ 
and $B$. Thus it follows from our previous considerations that the solutions of $P_{34}$ are meromorphic for any choice of parameters.

Let us demonstrate how to obtain the Bäcklund transformation for $P_{34}$ by using equation $P_{2}$. For $P_{2}$ if $f=f(z)$ is a solution of $P_{2}$ with the parameter $\alpha$, then the function

$$
f_{1}(z)=-f+(\alpha \varepsilon-1 / 2) /\left(f^{\prime}-\varepsilon f^{2}-\varepsilon z / 2\right), \quad \varepsilon^{2}=1,
$$

is a solution of equation $P_{2}$ with the parameter $\alpha-\varepsilon$. Using the Hamiltonian system, we obtain that if $f=f(z)$ is a solution of $P_{34}$ with the parameters $A=(2 \alpha+1)^{2} / 4$, $B=1$, then the functions

$$
f_{1}(z)=\frac{(2 \alpha+1)^{2}+8 z f^{2}-8 f^{3}-4 f^{\prime}-8 \alpha f^{\prime}+4 f^{\prime 2}}{8 f^{2}}
$$

and

$$
f_{2}(z)=\frac{(2 \alpha+1)^{2}+8 z f^{2}-8 f^{3}+4 f^{\prime}+8 \alpha f^{\prime}+4 f^{\prime 2}}{8 f^{2}}
$$

are the solutions of $P_{34}$ with the parameters

$$
A_{1}=(2 \alpha-1)^{2} / 4, \quad B_{1}=1
$$

and

$$
A_{2}=(2 \alpha+3)^{2} / 4, \quad B_{2}=1,
$$

respectively.

When $A=1, B=1$ equation $P_{34}$ has a one-parameter family of solutions

$$
\left(f^{\prime}\right)^{2}-2 f^{\prime}-2 f^{3}+2 z f^{2}+1=0 .
$$

It can be obtained from the Riccati solution $f^{\prime}=f^{2}+z / 2$ of $P_{2}$ with $\alpha=1 / 2$ by using the Hamiltonian system. [20].

Equation $\boldsymbol{P}_{\mathbf{4}, \mathbf{3 4}}$. It is convenient to treat equations $P_{4}$ and $P_{34}$ simultaneously

As we have already mentioned, in case $(C 2)$, putting $\alpha=-\beta / 2, \beta=b^{3}, \gamma=2 d^{4}$ in (7) we obtain that $2 d f(t)$, where $t=\left(z+b^{3} /\left(4 d^{4}\right)\right) d$ is a solution of $P_{4}(\tilde{\alpha}, \tilde{\beta})$ with parameters $\tilde{\alpha}=b^{6} d^{-6} / 16=\beta^{2} /\left(4 \sqrt{2} \gamma^{3 / 2}\right)$ and $\tilde{\beta}=\beta$ [20]. Clearly, by this change of variables one can find the Bäcklund transformations, one-parameter and rational solutions for special values of the parameters of $P_{4,34}(-\beta / 2, \beta, \gamma)$ coming from the corresponding transformations and solutions of $P_{4}[8]$.

By direct calculations we get that in case $(\mathrm{C} 1)$ the equation $P_{4,34}$ admits a oneparameter family of solutions of the form

$$
f^{\prime}(z)=\sqrt{2 \gamma} \delta f(z)^{2}+\frac{\beta+2 \gamma z}{\sqrt{2 \gamma} \delta} f(z)+\sqrt{\alpha} \varepsilon,
$$

where $\varepsilon^{2}=\delta^{2}=1, \gamma \neq 0$ and the parameters are related by

$$
\beta^{2}+4 \sqrt{2 \gamma} \gamma \delta(1+\sqrt{\alpha} \varepsilon)=0 .
$$

From equation $P_{34}$ we can get that the solutions of

$$
\left(f^{\prime}\right)^{2}+2 f^{\prime}-2 \beta f^{3}-2 \beta z f^{2}+1=0
$$

are also solutions of $P_{4,34}(1, \beta, 0)$. 
The Bäcklund transformation for $P_{4,34}$ in case (C2) can be obtained as follows. If $f(z)$ is a solution of $P_{4,34}(\alpha, \beta, \gamma)$, where $\alpha=\left(3 \sqrt{2} \beta^{2}+8 \delta \sqrt{\gamma} \gamma\right)^{2} /\left(576 \gamma^{3}\right)$ and $\delta^{2}=1$, then the function

$f_{1}(z)=-\left(f^{\prime}(z)+\sqrt{2 \gamma} \delta f(z)^{2}+\frac{(\beta+2 \gamma z)}{\sqrt{2 \gamma} \delta} f(z)+\frac{3 \sqrt{2} \beta^{2}+8 \sqrt{\gamma} \gamma \delta}{24 \sqrt{\gamma} \gamma \delta}\right) /(2 \sqrt{2 \gamma} \delta f(z))$

is a solution of $P_{4,34}\left(\alpha_{1}, \beta, \gamma\right)$ with

$$
\alpha_{1}=\alpha-\beta^{2} /(3 \delta \sqrt{2 \gamma} \gamma)=1 / 9+\beta^{4} /\left(32 \gamma^{3}\right)-\beta^{2} /(6 \delta \sqrt{2 \gamma} \gamma)
$$

Acknowledgements. GF is supported by the National Science Center grant 2011/ 03/B/ST1/00330. We thank the referee for useful comments and suggestions.

\section{References}

[1] Clarkson, P. A.: Painlevé transcendents. - NIST Digital Library of Mathematical Functions, http://dlmf.nist.gov/32.

[2] Clarkson, P. A., N. Joshi, and A. Pickering: Bäcklund transformations for the second Painlevé hierarchy: a modified truncation approach. - Inverse Problems 15, 1999, 175-187.

[3] Clunie, J.: On integral and meromorphic functions. - J. London Math. Soc. 37, 1962, $17-27$.

[4] Gambier, B.: Sur les équations différentielles du second ordre et du premier degré dont lâ intégrale générale est à points critiques fixés. - Acta Math. 33, 1909, 1-55.

[5] Gol'dberg, A. A., A. E. Eremenko, and M. L. Sodin: Exceptional values in the sense of R. Nevanlinna and in the sense of V. Petrenko, I. - Teor. Funkts., Funkts. Anal. Prilozh. 47, 1987, 41-51. Engl. transl.: J. Sov. Math. 48:6, 1990, 648-655.

[6] Gol'dberg, A. A., A. E. Eremenko, and M. L. Sodin: Exceptional values in the sense of R. Nevanlinna and in the sense of V. Petrenko, II. - Teor. Funkts., Funkts. Anal. Prilozh. 48, 1987, 58-70. Engl. transl.: J. Sov. Math. 49:2, 1990, 891-899.

[7] Govorov, N. V.: On the Paley hypothesis. - Funkts. Anal. Prolozhen. 3:2, 1969, 41-45 (in Russian).

[8] Gromak, V., I. Laine, and S. Shimomura: Painlevé differential equations in the complex plane. - de Gruyter Stud. Math. 28, Walter de Gruyter, Berlin, 2002.

[9] Hayman, W. K.: Meromorphic functions. - Oxford, Clarendon Press, 1964.

[10] Hinkkanen, A., and I. Laine: Solutions of the first and second Painlevé equations are meromorphic. - J. Anal. Math. 79, 1999, 345-377.

[11] InCE, E. L.: Ordinary differential equations. - London, Longmans Green, 1927.

[12] Imasaki, K., H. Kimura, S. Shimomura, and M. Yoshida: From Gauss to Painlevé. A modern theory of special functions. - Aspects Math. E16, Vieweg \& Sohn, Braunschweig, 1991.

[13] Kiessling, H.: Zur Werteverteilung der Lösungen algebraischer Differentialgleichungen. Ph.D. thesis, Berlin, 1996.

[14] Laine, I.: Nevanlinna theory and complex differential equations. - Berlin, New York, De Gruyter, 1993.

[15] Marchenko, I. I., and A. I. ShCherba: On the magnitudes of deviations of meromorphic functions. - Mat. Sb. 181, 1990, 3-24 (in Russian). Engl. transl.: Math. USSR Sbornik, 69:1, $1991,1-24$.

[16] Marchenko, I. I.: An analogue of the second main theorem for the uniform metric. - Mat. Fizika, Analiz, Geometria 5:3/4, 1998, 212-227 (in Russian).

[17] Mohon'ko, A.Z., and V.D. Mohon'ko: Estimates of the Nevanlinna characteristics of certain classes of meromorphic functions, and their applications to differential equations. Sibirsk. Mat. Zh. 15, 1974, 1305-1322 (in Russian). 
[18] Mohon'Ko, V. D.: Magnitudes of deviations for the transcendental solution of a differential equation. - Differ. Uravn. 14:7, 1978, 1328-1331 (in Russian).

[19] Nevanlinna, R.: Eindeutige Analytische Funktionen. - Springer-Verlag, Berlin, 1970.

[20] Ohyama, Y., and S. Okumura: A coalescent diagram of the Painlevé equations from the viewpoint of isomonodromic deformations. - J. Phys. A: Math. Gen. 39, 2006, 12129.

[21] Окамото, K.: Studies on the Painlevé equations. III. Second and fourth Painlevé equations, $P_{I I}$ and $P_{I V}$. - Math. Ann. 275, 1986, 221-255.

[22] Petrenko, V. P.: The growth of meromorphic functions of finite lower order. - Izv. Akad. Nauk SSSR 33:2, 1969, 414-454 (in Russian).

[23] Schubart, H.: Zur Wertverteilung der Painlevéschen Transzendenten. - Arch. Math. (Basel) 7, 1956, 284-290.

[24] Schubart, H., and H. Wiтtich: Über die Lösungen der ersten beiden Painlevéschen Differentialgleichungen. - Math. Z. 66, 1957, 364-370.

[25] Shimomura, S.: Proofs of the Painlevé property for all Painlevé equations. - Japan J. Math. 29, 2003, 159-179.

[26] Shimomura, S.: The first, the second and the fourth Painlevé transcendents are of finite order. - Proc. Japan Acad. Ser. A 77, 2001, 42-45.

[27] Shimomura, S.: Growth of the first, the second and the fourth Painleve transcendents. Math. Proc. Cambr. Philos. Soc. 134:2, 2003, 259-269.

[28] Steinmetz, N.: Zur Wertverteilung der Lösungen der vierten Painlevéschen Differentialgleichung. - Math. Z. 181, 1982, 553-561.

[29] Steinmetz, N.: On Painlevé equations I, II and IV. - J. Anal. Math. 82, 2000, 363-377.

[30] Steinmetz, N.: Value distribution of the Painlevé transcendents. - Israel J. Math. 128, 2002, $29-52$.

Received 16 September 2015 • Accepted 15 January 2016 